\title{
Functional characterization of mungbean CONSTANS-LIKE genes reveals a key role for CONSTANS-LIKE 2 in the control of flowering time in $A$. thaliana under short-day conditions
}

Chenyang Liu

Qingdao Agricultural University

Qianqian Zhang

Qingdao Agricultural University

Hong Zhu

Qingdao Agricultural University

Chunmei Cai

Qingdao Agricultural University

Shuai Li ( $D$ li2014shuai@qau.edu.cn )

Qingdao Agricultural University https://orcid.org/0000-0002-6905-2349

Research article

Keywords: Mungbean, Flowering time, CONSTANS, VrCOL2, Genome wide

Posted Date: July 24th, 2020

DOI: https://doi.org/10.21203/rs.3.rs-24842/v2

License: (c) (i) This work is licensed under a Creative Commons Attribution 4.0 International License.

Read Full License 


\section{Abstract}

Background: CONSTANS-LIKE (COL) genes play important roles in the regulation of plant growth and development, and they have been analyzed in many plant species. However, few investigations have examined $C O L$ genes in mungbean (Vigna radiata).

Results: In this study, we identified and characterized a total 14 of $\mathrm{VrCOL}$ genes from mungbean, which distributed on 7 of the 11 mungbean chromosomes. Based on their conserved domains, VrCOLs were clustered into three groups (I, II and III), which contained 4, 5 and 5 members, respectively. The gene structures and conserved motifs of the $\mathrm{V} r C O L$ genes were analyzed, and two duplicated gene pairs, VrCOL 1/VrCOL2 and VrCOL8/VrCOL9, were identified. A total of $82 \mathrm{cis}$-acting elements were found in the $\mathrm{VrCOL}$ promoter regions, and the numbers and types of cis-acting elements in each $\mathrm{VrCOL}$ promoter region differed. As a result, the expression patterns of VrCOLs varied in different tissues, and under long day and short day conations throughout the day. Among these $\mathrm{VrCOL}$ genes, $\mathrm{VrCOL} 2$ showed a close phylogenetic relationship with Arabidopsis thaliana (A. thaliana) $\mathrm{CO}$ and displayed daily oscillations in expression under short day conditions but not long day conditions. In addition, overexpression of VrCOL2 accelerated flowering in $A$. thaliana under short day conditions by activating the expression of flowering time gene AtFT and AtTSF.

Conclusion: Overall, we identified $14 \mathrm{VrCOL}$ genes from mungbean using genome-wide identification. Characteristics and transcription pattern analysis of $\mathrm{VrCOL}$ genes revealed their important roles in plant growth and development, and our results suggested that $\operatorname{VrCOL} 2$ regulate flowering time under short day conditions in A. thaliana. Our study lays the foundation for further dissection of $\mathrm{VrCOL}$ gene functions.

\section{Background}

Flowering time is a key factor that influences crop growth and development, and crops achieve higher yields when they flower at the correct time. To regulate flowering time, crops sense the interactions between endogenous and environmental factors to determinate the transition from vegetative to reproductive growth [1-4]. Several functional pathways have been identified that regulate the switch from vegetative to reproductive development. These include the photoperiodic, vernalization, ambient temperature, plant hormone, and autonomous flowering pathways [1, 4-10]. Within these pathways, a number of genes have been identified as involved in flowering time regulation, including CONSTANS-LIKE (COL) genes, phosphatidyl ethanolamine-binding protein (PEBP) genes and several members of MADSBox gene family [1-2, 11-19].

$\mathrm{COL}$ genes belong to the zinc-finger transcription factor family and play central roles in plant growth and development $[11,20]$. COL proteins are identified based on their conserved structure, which includes one or two BBX (B-Box) domains and one CCT (CONSTANS, CO-like, and TIMING of CAB1) domain [11, 20]. The BBX domain can be further divided into two types, B-Box1 and B-Box2, which are recognized by their consensus sequences and the distances between their zinc-binding residues, which are considered to be 
involved in protein-protein interactions [20]. The CCT domain has important functions in transcriptional regulation and nuclear protein transport [11,20-22]. The COL proteins are classified into three classes based on the number and type of their conserved domains. Class I and II have two distinct BBX domains and one CCT domain, whereas class III has only one BBX and one CCT domain. Classes I, II, III contain 6, 7 and 4 members in Arabidopsis thaliana (A. thaliana), respectively. In addition, several COL proteins contain valine-proline (VP) motifs in their $C$ termini $[11,20]$.

Among these $C O L$ members, AtCO (AtBBX1) and its homologs are well-studied in many plant species [11, 20,23-25]. AtCO is expressed in a rhythmic manner and coordinates light pathway and circadian clock signal inputs in A. thaliana [26-29]. Thus, AtCO plays an important role in the regulation of flowering time via the photoperiod-dependent pathway. Atco mutants exhibit delayed flowering time under long day conditions (LD), but under short day conditions (SD), their flowering times are similar to those of wild-type plants. By contrast, AtCO overexpression plants show early flowering time under both LD and SD conditions $[11,20]$. The AtCO protein binds to cis-acting elements in the promoter region of the flowering activator FLOWERING LOCUS T (AtFT) to active AtFT expression. Moreover, AtCO is regulated by many flowering factors, such as AtGI (GIGANTEA), AtCDF1 (CYCKLING DOF FACTOR 1) and AtFKF1 (FLAVIN BINDING, KELCH REPEAT, F-BOX1) [30-31]. OsHd1 (Heading date 1), the AtCO ortholog in rice, accelerates flowering under SD but delays flowering time under LD conditions through the regulation of the AtFT orthologs OsHd3a (Heading date 3a) and OsRFT1 (RICE FLOWERING LOCUS T1) [32-34]. The soybean AtCO orthologs GmCOL 1, GmCOL2, GmCOL3 and GmCOL4 can complement the late flowering phenotype of Atco mutants [35]. In addition to their functions in flowering time and circadian clock regulation, some $\mathrm{COL}$ proteins are also involved in abiotic or biotic stress responses, root development and stomatal opening $[11,20]$.

Mungbean is a diploid legume crop, and its seeds contain proteins and nutrients that are essential for human nutrition [36]. The cultivated mungbean is considered to have been domesticated in India, from which it then spread to other areas [37]. Mungbean is considered to be a SD crop, and the flowering time is a critical factor influencing its production [38-40]. Mungbean plants produce a large number of flowers, but only a few set pods. Approximately $70-90 \%$ of the flowers are shed, which mainly occurs in later formed flowers of the racemes [41-42]. Thus, it is suggested that the prevention of late flowering is an important way to increase mungbean yield [42-44]. The sequencing of mungbean genome provides genetic resources for the investigation of gene functions [45], and the investigation of mungbean flowering time genes can therefore provide essential information for further modification of mungbean cultivars to increase yield. Until now, limited information about gene functions has been identified in mungbean flowering time regulation. In this study, we identified mungbean $\mathrm{COL}$ genes and investigated their characteristics, including chromosomal distributions, gene structures, cis-acting elements and gene expression patterns. We also analyzed the functions of $\mathrm{VrCOL} 2$ in the regulation of flowering time under SD conditions. Our findings will provide useful information for further characterization of mungbean $C O L$ gene functions. 


\section{Results}

\section{Identification of $\operatorname{VrCOL}$ genes in mungbean}

To search for mungbean $\mathrm{VrCOL}$ genes, we used the amino acid sequences of AtCO and AtCOL proteins as blast queries against the mungbean genome database and the NCBI database. The conserved BBX and CCT domains in each candidate gene were confirmed using Pfam and InterPro, and a total of $14 \mathrm{VrCOL}$ members were identified in the mungbean genome. Multiple characteristics of the $\mathrm{V} r C O L$ members were analyzed based on their genomic and protein sequences (Table 1). The genomic lengths of $\mathrm{VrCOL}$ genes ranged from 1,506 (XP_014502470) to 14,007 bp (XP_014523701), the CDS lengths ranged from 933 (XP_014502470) to 1,329 bp (XP_022637309), and the amino acid numbers ranged from 310 to 442 . The isoelectric points of VrCOL proteins varied from 4.86 (XP_014523701) to 9.22 (XP_014523547), and their molecular weights ranged from 33,756.82 (XP_014502470) to 48,806.9 (XP_022637309) Da. The GC content, which influences gene stability to some degree, ranged from $34.64 \%$ to $50.39 \%$, and twelve of the fourteen $\operatorname{VrCOL}$ genes had lower than $50 \%$ GC content (Table 1).

\section{Phylogenetic and classification analysis of VrCOL proteins}

To analyze the evolutionary relationships among the $\mathrm{V} r C O L$ genes and obtain information from wellstudied $\mathrm{CO}$ homologs in other species, a phylogenetic tree was constructed using $17 \mathrm{~A}$. thaliana, 26 soybean, 11 Medicago, 16 rice, 18 maize and 14 mungbean CO and COL proteins [11, 35, 46-47]. The VrCOL genes were named from $\operatorname{VrCOL} 1$ to $\operatorname{VrCOL} 13$ based on their phylogenetic relationships with the soybean orthologous genes (Fig. 1, Table 1). Among these VrCOL members, VrCOL 1 and VrCOL2 showed close relationships to $A$. thalianaAtCO, soybean GmCOL1a, GmCOL 1b, GmCOL2a, and GmCOL2b and rice OsHd1 (OsCOL-A), all of which have documented roles in the regulation of flowering time [11, 20,35]. This result suggests that $\operatorname{VrCOL} 1$ and $\operatorname{VrCOL} 2$ may play critical roles in the flowering time regulation of mungbean.

The COL proteins were classified into three groups based on differences in the numbers and types of conserved BBX and CCT domains $[11,20]$. We analyzed the $\operatorname{VrCOL~BBX}$ and CCT domains and found two distinct BBX domains (BBX1 and BBX2) and one CCT domain (Additional file 1). The sequence logos of the BBX1 ( $\left.\mathrm{CX}_{2} \mathrm{CX}_{8} \mathrm{CX}_{4} \mathrm{AXLCX}{ }_{2} \mathrm{CDX}{ }_{3} \mathrm{HX}{ }_{8} \mathrm{HXR}\right), \mathrm{BBX} 2\left(\mathrm{CX}_{2} \mathrm{CX}_{4} \mathrm{AX}_{3} \mathrm{CX}_{7} \mathrm{CX}_{2} \mathrm{CDX}_{3} \mathrm{HX}{ }_{8} \mathrm{H}\right)$ and CCT $\left(\mathrm{RYX}_{2} \mathrm{KX}_{3} \mathrm{RX}_{3} \mathrm{KX}{ }_{2} \mathrm{RYX}_{2} \mathrm{RKX}_{2} \mathrm{AX}_{2} \mathrm{RXR}\right.$ ) domains were determined using WebLogo (Fig. 2, Additional file 1). Nine $\mathrm{V} r C O L$ proteins contained one BBX1, one BBX2 and one CCT domain, and five $\mathrm{V} r C O L$ proteins contained one BBX1 and one CCT domain (Fig. 3). The VrCOL proteins were further classified into three groups based on differences in these conserved domains (Fig. 1 and 3). Classes I, II and III contained 4, 5 and $5 \mathrm{VrCOL}$ members, respectively. The BBX1 and BBX2 domains were located close to one another in the class I and II genes (Fig. 3). Most of the VrCOL genes from the same class were clustered into the same clade in the phylogenetic tree, with the exception of class III member VrCOL10, which showed a closer relationship with class II members (Fig. 1 and 3). 


\section{Gene structures and conserved motifs of the $\operatorname{VrCOL}$ genes}

To investigate the gene structures of the $\mathrm{VrCOL}$ genes, we downloaded their genomic and CDS sequences from the $\mathrm{NCBI}$ and analyzed them using the GSDS program [48]. All the $\mathrm{VrCOL}$ members contained 5' UTR and 3' UTR regions. Their exon numbers ranged from 2 to 6 , and their intron numbers ranged from 1 to 6 . Most group I and III VrCOL members contained two exons and one intron, suggesting conserved functions of the genes within each group. An exception was class III member VrCOL 10, which contained 6 exons and 6 introns and had a close relationship with group II members (Fig. 3-4). By contrast, group II members contained various numbers of exons ( 3 to 5 ) and introns ( 2 to 5 ), suggesting potential functional diversity among these genes (Fig. 4). To further investigate the conservation and diversity of $\mathrm{VrCOL}$ protein structures, we analyzed putative protein motifs in the VrCOLs. A total of 17 distinct motifs were identified, and all VrCOL proteins contained motifs 1 and 2, which appeared to represent the conserved CCT and BBX1 domains, respectively (Fig. 4, Additional file 2). Most members of the same group shared some conserved motifs. For example, group I proteins shared motifs 1, 2, 3, 9 and 16, group II members shared motifs 1, 2, 3, and 5, and most group III members shared motifs 1, 2, 4, 8, 12, and 13 (except for VrCOL10) (Fig. 4).

\section{Chromosomal distribution and duplication analysis of VrCOL genes}

Plant $C O L$ genes evolved from several common original genes, and the chromosomal locations of $C O L$ genes can represent the alteration of gene distributions during evolution. To visualize the chromosomal locations of the $\mathrm{VrCOL}$ genes, we mapped them to their physical positions in the mungbean genome. $\operatorname{VrCOL} 7 b$ was discarded due to a lack of related chromosome information. Seven of the fourteen $\mathrm{VrCOL}$ genes were located on the positive strand. Seven of the eleven mungbean chromosomes contained VrCOL genes, with the exception of chromosomes 2, 9, 10 and 11 (Fig. 5, Table 1). Chromosome 5 contained the most number of $\mathrm{VrCOL}$ genes (three), followed by chromosomes 1, 4, 7 and 8, with two genes on each. In addition, most of the $\mathrm{VrCOL}$ genes were located on the relatively long chromosomes (1, 5, 6, 7 and 8). Only three members ( $\operatorname{VrCOL1}, \operatorname{VrCOL} 11$ and $\operatorname{VrCOL13)}$ were located on the relatively short chromosomes 3 and 4 (Fig. 5).

Mungbean has experienced one round of whole-genome duplication that produced many duplicated gene pairs $[45,49]$. To investigate the evolutionary relationships among the $\operatorname{VrCOLs}$, we searched for duplicated gene pairs among them. Two interchromosomal duplication events were identified in chromosomes 1, 4, 5 and 6, including the duplicated gene pairs VrCOL1/VrCOL2 and VrCOL8/VrCOL9 (Fig. 6). The duplicated genes were clustered together in the phylogenetic tree (Fig. 3). All the duplicated genes contained one BBX1, one BBX2 and one CCT domain and belonged to groups I and II, no duplicated gene pairs were found in group III. The duplicated genes $\operatorname{VrCOL} 1$ and $\mathrm{VrCOL} 2$ showed similar exon-intron organization and similar motifs, as did $\operatorname{VrCOL} 8$ and $\operatorname{VrCOL9}$ (Fig. 4), indicating that the duplicates may share similar functions. 


\section{Cis-acting element analysis of the VrCOL promoter regions}

To predict the potential expression response of $\mathrm{VrCOL}$ genes, we investigated the cis-acting elements in their promoters using PantCARE [50]. A total of 82 cis-acting elements were found across the $14 \mathrm{VrCOL}$ promoter regions ( $2 \mathrm{~kb}$ upstream of the initiation codon) (Additional file 3). Forty-five of them had predicted functions, including six development-related elements, four environmental-stress-related elements, three site-binding-related elements, nine hormone-responsive elements, three promoter-related elements and twenty light-responsive elements (Table 2, Additional file 3). The various VrCOL promoter regions had different numbers and types of $c i s$-acting elements, highlighting the functional diversity of these genes. All VrCOL promoters contained hormone-responsive elements, light-responsive elements and promoter related elements, and light-responsive elements were the most abundant element in each $\mathrm{VrCOL}$ promoter, with the exception of $\mathrm{VrCOL8}$ (Table 2), indicating that $\mathrm{VrCOL}$ genes may play critical roles in light-dependent signaling pathways. Environmental-stress-related elements were the most abundant element in the $\mathrm{VrCOL8}$ promoter (nine elements), indicating that $\mathrm{VrCOL} 8$ may function in stress response (Table 2). All the VrCOL genes contained the promoter-related elements CAAT-Box and TATA-Box, which are basic promoter components. Thirteen of the $14 \mathrm{VrCOLs}$ contained the hormone-responsive elements CGTCA-motif and TGACG-motif and the light-responsive element Box 4 (Additional file 3), suggesting potential functions of these genes in related signaling pathways.

\section{Transcription patterns of $\operatorname{VrCOL}$ genes in different tissues}

To shed light on the potential functions of $\mathrm{VrCOL}$ genes during plant development, we analyzed the expression of $V r C O L$ genes in different tissues, including roots, nodule roots, shoot apices, stems, leaves, flowers, pods and seeds. VrCOL genes showed distinct expression patterns in different tissues (Fig. 7). For example, VrCOL3 was highly expressed in all the tested tissues, whereas $\mathrm{VrCOL} 2$ and $\mathrm{VrCOL} 7 \mathrm{a}$ showed low expression in most tissues. Some genes were expressed at high levels in specific tissues, suggesting that they may have critical functions in these tissues. For example, VrCOL6 showed high expression in leaves but low expression in nodule roots and roots.

Duplicated genes may retain some common functions and evolve some new functions [51-52]. To investigate the conservation and diversity of duplicated genes, we also analyzed their tissue-specific expression patterns. VrCOL 1 and $\operatorname{VrCOL} 2$ differed in their expression levels across all the tissues we examined, indicating that they had undergone functional divergence. VrCOL 8 and $\operatorname{VrCOL} 9$ showed similar expression levels in roots and nodule roots, but they exhibited different expression levels in other tissues (Fig. 7, Additional file 4).

\section{Diurnal rhythm of $\operatorname{VrCOL}$ gene expressions}

In $A$. thaliana, the expressions of $C O, C O L 1$ and $C O L 2$ are regulated by the circadian clock and show diurnal oscillations $[11,53]$. We therefore investigated whether $\mathrm{VrCOL}$ genes exhibited diurnal expression 
rhythms in mungbean leaves under LD and SD conditions. Gene expression analysis revealed that VrCOL4, VrCOL6, VrCOL12, and VrCOL 13 showed daily oscillations under both LD and SD conditions, while VrCOL 1, VrCOL2, VrCOL5, VrCOL7a, VrCOL7b, VrCOL 10 and VrCOL 11 only showed daily oscillations under SD conditions, but not LD conditions (Fig. 8). The duplicated genes VrCOL8 and VrCOL 9 exhibited similar expression patterns under both LD and SD conditions, while $\operatorname{VrCOL} 1$ and $\operatorname{VrCOL} 2$ showed distinct expression patterns under both LD and SD throughout the day (Fig. 8).

\section{Overexpression of $\mathrm{VrCOL2}$ accelerates flowering in $A$. thaliana under SD conditions}

VrCOL 1 and $\operatorname{VrCOL2}$ displayed close phylogenetic relationships with AtCO (Fig. 1), and the amino acid sequences of $\mathrm{VrCOL} 1$ and $\mathrm{VrCOL} 2$ showed $49.35 \%$ and $50.93 \%$ similarities with AtCO, respectively, thus VrCOL2 was selected for further analysis. To investigate the potential functions of $\mathrm{VrCOL} 2$ in flowering time regulation, $\mathrm{VrCOL} 2$ was transformed into $A$. thaliana under the control of the $35 \mathrm{~S}$ promoter. The empty vector was also transformed into $A$. thaliana, and the transgenic plants showed no difference with wild type under both LD and SD conditions (Additional file 5). The VrCOL2 transgenic A. thaliana lines showed high levels of $\mathrm{VrCOL} 2$ expression (Additional file 6). The $\mathrm{VrCOL2}$ overexpression lines exhibited similar flowering time to wild-type plants under LD conditions but exhibited earlier flowering time than wild-type plants under SD conditions (Fig. 9), indicating that VrCOL2 regulates flowering time under a photoperiod-dependent pathway.

AtFT and AtTSF accelerate flowering and are regulated by $A t C O$ in $A$. thaliana [11, 20], and we therefore investigated the expression of AtFT and AtTSF in wild-type and VrCOL2 transgenic plants under LD and SD conditions. AtFT and AtTSF showed similar expression levels in VrCOL2 transgenic and wild-type plants under LD conditions. By contrast, AtFT and AtTSF showed higher expression levels in VrCOL2 transgenic plants than in wild-type plants under SD conditions (Fig. 9). In addition, the expressions of two mungbean FT and TSF homologous genes (XP_014496932, XP_014497364), which contained CORE cisacting elements in their promoter regions, increased in VrCOL2 transgenic hair roots under SD conditions, but not LD condition (Additional file 7). These results further support the conclusion that $\operatorname{VrCOL} 2$ is involved in flowering time regulation under SD conditions.

\section{Discussion}

In recent decades, the investigation of $\mathrm{CO}$ and $\mathrm{COL}$ genes in many plant species has greatly increased our knowledge about the molecular mechanisms of flowering time regulation, stress response and root development $[11,20]$. Mungbean is a globally important legume crop, and the mechanisms of its flowering time regulation are still largely unknown. In this study, we identified and characterized $14 \mathrm{VrCOL}$ genes from the mungbean genome and investigated the function of $\mathrm{VrCOL2}$ in flowering time regulation. 
The A. thaliana, soybean, Medicago and mungbean genomes contained 17, 26, 11 and $14 \mathrm{CO}$ and $\mathrm{COL}$ members, respectively (Fig. 1) [35, 54], and their genome sizes are $125 \mathrm{Mb}$ [55], $1100 \mathrm{Mb}$ [56], $500 \mathrm{Mb}$ [57] and $579 \mathrm{Mb}$ [45], respectively. Thus, genome size has no direct relationship with the number of $\mathrm{COL}$ genes in plants. Soybean has undergone two rounds of whole-genome duplication, whereas mungbean has experienced only one such duplication $[45,56]$. As a result, the $C O L$ gene number in mungbean is approximately half that of soybean. Seven of the eleven (63.6\%) mungbean chromosomes (Fig. 5), seven of the eight (87.5\%) Medicago chromosomes and sixteen of the twenty $(80.0 \%)$ soybean chromosomes contained $\mathrm{COL}$ genes [35,54], indicating that the distribution of $\mathrm{COL}$ genes has changed much during evolution in legumes. The $C O L$ genes were clustered into three groups based on their conserved domains, and most of $\mathrm{VrCOL}$ genes in each group were clustered into the same clade in the phylogenetic tree, with the exception of the group III member VrCOL10, which contained one BBX and one CCT domain and showed a close relationship with group II members (Fig. 1 and 3). VrCOL10 protein lacked motifs 4, 8, 12, and 13, which were found in all other VrCOL group III members, but it did contain motifs 3 and 5 , which were found in all $\mathrm{VrCOL}$ group II members (Fig. 4), suggesting that $\mathrm{VrCOL} 10$ may derive from a group II ancestor and that one BBX domain may have been lost during evolution.

Plant genome evolution produces many duplicated gene pairs and provides resources for new gene functions [51]. Two duplicated gene pairs, VrCOL1/VrCOL2 and VrCOL8/VrCOL9 (Fig. 6), were found among the mungbean $\mathrm{VrCOLs}$. The duplicated genes showed close relationships in the phylogenetic tree and contained similar motifs (Fig. 3-4), indicating that they evolved from the same origin and likely shared similar functions. However, the duplicated gene pairs contained different numbers and types of cis-acting elements in their promoter regions and exhibited different expression levels in some tissues (Fig. 7, Table 2), suggesting that they might have evolved novel functions compared with their original gene. For example, $\operatorname{VrCOL} 8$ and $\operatorname{VrCOL9}$ shared similar numbers of several cis-acting elements in their promoter regions, including promoter-related elements and site-binding related elements, but differed in the numbers of development-related elements, environmental-stress-related elements, hormoneresponsive elements and light-responsive elements (Table 2, Additional file 3). VrCOL8 and VrCOL9 showed similar expression levels in roots and nodule roots, but their expression differed in flowers, pods, leaves, seeds, stems and shoot apices (Fig. 7, Additional file 4). This result suggests that they may have retained some common functions from the original gene in roots and nodule roots but evolved novel functions in other tissues.

The expression of $\mathrm{VrCOL}$ genes in different tissues provides clues to their potential functions, and many VrCOL genes (such as VrCOL6 and VrCOL 12) showed tissue-specific expression patterns (Fig. 7). However, several $\mathrm{V} C \mathrm{COL}$ genes showed low expression levels in all tissues tested, despite the fact that their promoter regions contained many cis-acting elements, including VrCOL2, VrCOL7a and VrCOL 10 (Fig. 7, Table 2, Additional file 3). Gene expression is influenced by many factors. For example, many circadian clock and flowering time regulation genes are controlled by photoperiod. Their expression changes under different photoperiods and during the day and night $[1,4,6,53]$. For example, VrCOL2 appeared to be a daily oscillation gene whose expression changed during the day under SD conditions but showed low expression throughout the day under LD conditions (Fig. 8). The different field-grown 
mungbean tissues were collected in the afternoon under relatively LD conditions in July, and that may explain why VrCOL2 showed low expression levels in the tissue expression analysis (Fig. 7).

$\mathrm{CO}$ and $\mathrm{CO}$-homologous genes, such as $\mathrm{OsHd1}$, play critical roles in flowering time regulation $[11,20]$. VrCOL2 showed higher expression levels at the stages of during flowering, and after flowering, than that at the stage of before flowering, indicating that $\mathrm{VrCOL2}$ played an important role in flowering time regulation (Additional file 8). VrCOL2 showed close relationships with A. thaliana CO, soybean GmCOL 1a, GmCOL 1b, GmCOL2a and GmCOL2b and rice OsHd1 (OsCOL-A), and accelerated flowering under SD but not LD conditions in transgenic $A$. thaliana lines (Fig. 9). AtCO regulates AtFT and AtTSF to accelerate flowering [26-29], and the expression of AtFT and AtTSF increased in VrCOL2 transgenic A. thaliana lines under SD but not LD conditions (Fig. 9), indicating that VrCOL2 regulates downstream genes via photoperiod-dependent pathways. In addition, AtCO protein accumulation is also regulated by circadian clock. AtCO mRNA abundance is highly expressed from late afternoon to the dawn, but AtCO protein only accumulates in the late afternoon under long day conditions [27, 58-60]. Although $\mathrm{VrCOL2}$ is controlled by $35 \mathrm{~S}$ promoter and can be expressed under both LD and SD conditions, the accumulation of VrCOL2 proteins might be low under LD condition, which might be the reason why $\operatorname{VrCOL2}$ had no effect on flowering time under LD conditions. Overexpression of AtCO accelerates flowering under both LD and SD conditions [11, 20], while in rice OsHd1 accelerates flowering under SD but delays flowering time under LD conditions [32-34]. Mungbean [38-39] and rice are short day plants, and $A$. thaliana is a long day plant, which might be the reason why $\mathrm{CO}$ homologous genes have different functions in different plant species. These results suggest that $\mathrm{CO}$ and its homologs are involved in flowering time regulation under photoperiod-dependent pathways and have distinct roles in different plant species. Thus, in summer LD conditions, the expression of VrCOL2 may be low and have little effect on the acceleration of flowering. In the autumn, as days become shorter, the expression of $\mathrm{VrCOL} 2$ may increase and accelerate mungbean flowering. In addition, $\operatorname{VrCOL} 1$ and $\mathrm{VrCOL} 2$ form a duplicated gene pair and show a close relationship with one another (Fig. 1 and 6), indicating that $\operatorname{VrCOL} 1$ may have similar functions to $\operatorname{VrCOL} 2$ in flowering time regulation. Much more work is needed to fully elucidate the mechanisms by which $\operatorname{VrCOL} 2$ affects flowering time and circadian clock regulation in mungbean.

\section{Conclusion}

In this study, we identified and characterized $14 \mathrm{VrCOL}$ genes from mungbean genome using genomewide analysis, and many characteristics of these $\mathrm{VrCOL}$ genes were investigated, including chromosomal distributions, sequence logos, classifications, phylogenetic relationships, gene structures, conserved motifs, duplicated gene pairs, cis-acting elements, and expression profiles. Among these $\mathrm{VrCOL}$ genes, $\mathrm{VrCOL} 2$ showed a close relationship with AtCO and the expression of $\mathrm{VrCOL2}$ exhibited daily oscillations under SD conditions. Further investigation revealed that $\mathrm{VrCOL2}$ accelerated flowering under SD conditions by activating the expression of AtFT and AtTSF, but not LD conditions.

\section{Methods}




\section{Plant materials and growth conditions}

The reference mungbean genome variety VC1973A, stored at Seoul National University, was identified and supplied by Suk-Ha Lee at Seoul National University, Seoul, Korea [45], and the variety was used for all experiments in this study. Mungbean seeds were geminated in tap water for 1 day and then planted in soil-filled pots. Seedlings were grown in growth chambers with $16 \mathrm{~h} 25^{\circ} \mathrm{C}$ light/ $8 \mathrm{~h} 25^{\circ} \mathrm{C}$ dark and $10 \mathrm{~h}$ $25^{\circ} \mathrm{C}$ light/ $14 \mathrm{~h} 25^{\circ} \mathrm{C}$ dark cycles for LD and SD photoperiods, respectively. Leaves of 5-week old mungbean plants were sampled every 4 hours after lights-on and used to analyze the diurnal rhythm of gene expression. Multiple tissues were collected from field-grown mungbean plants sown in Qingdao, China, at the end of May, including roots, nodule roots, shoot apices, stems, leaves, flowers, pods and seeds. Tissues were collected in the afternoon, which might be ZT 10-12, in early July for gene expression analysis, and all samples were stored at $-80^{\circ} \mathrm{C}$ before RNA extraction. Moreover, mungbean leaves at three different stages, before flowering (S1), during flowering (S2, the first flower occurred), and after flowering ( $\mathrm{S} 3$, after the first flower occurred), were collected from field-grown plants for gene expression analysis. A. thaliana plants were grown in growth chambers with $16 \mathrm{~h} 23^{\circ} \mathrm{C}$ light $/ 8 \mathrm{~h} 21^{\circ} \mathrm{C}$ dark and $10 \mathrm{~h}$ $23^{\circ} \mathrm{C}$ light $/ 14 \mathrm{~h} 21^{\circ} \mathrm{C}$ dark cycles for LD and SD photoperiod treatments, respectively. Leaves of 3-weekold $A$. thaliana were collected for gene expression analysis.

\section{Identification of mungbean VrCOL members}

The amino acid sequences of $A$. thaliana $\mathrm{CO}$ and $\mathrm{COLs}$ were used as blast queries against the National Center for Biotechnology Information (NCBI) and mungbean genome database (http://plantgenomics.snu.ac.kr/mediawiki-1.21.3/index.php/Main_Page) [45] to search for mungbean $\mathrm{VrCOL}$ proteins. The presence of conserved BBX and CCT domains in candidate genes were confirmed using the Pfam database [61] and InterPro program with default parameters [62].

\section{Phylogenetic relationship analysis}

The amino acid sequences of $\mathrm{CO}$ and $\mathrm{COL}$ proteins from $A$. thaliana, soybean, Medicago, mungbean, rice and maize were aligned using ClustalW2 [63], and the resulting alignment was used to construct a phylogenetic tree in MEGA 7.0 using the Neighbor-Joining method with default parameters [64]. In addition, $\mathrm{VrCOL}$ proteins were aligned separately in ClustalW2 and used to construct a phylogenetic tree in MEGA7.0 with the Neighbor-Joining method.

\section{Chromosomal distribution and duplication analyses}

The physical positions of $\mathrm{VrCOL}$ genes were obtained from NCBI, and a chromosomal location map was constructed using Maplnspect software (Mike Lischke, Berlin, Germany). Duplicated gene pairs were 
identified using OrthoMCL software as described by Jin et al. [19,65]. The duplicated gene pairs were identified with amino acid sequences more than $60 \%$ similarity, and visualized using Circos software [66].

\section{Analyses of exon-intron organizations, conserved domains, sequence logos, protein motifs and cis-acting elements}

The genomic and CDS sequences of mungbean $\mathrm{V} r C O L$ genes were obtained from $\mathrm{NCBI}$ and used as inputs to the Gene Structure Display Server (GSDS) to analyze their gene structures [48]. The full-length amino acid sequences of $\mathrm{VrCOL}$ proteins were used to analyze the positions of the conserved BBX and CCT domains using the InterPro program [62]. The sequence logos of the conserved BBX1, BBX2 and CCT domains were analyzed using the WebLogo platform [67]. The conserved motifs present in the VrCOL proteins were identified using MEME tools, and the parameters of the optimum motif widths were 11-50 amino acid residues [68]. The cis-acting elements in each $\mathrm{VrCOL}$ promoter, $2 \mathrm{~kb}$ upstream of the initiation codon, were predicted by PlantCARE [50].

\section{Plasmid construction and plant transformation}

To investigate the functions of VrCOL2, a 35S: CDS-VrCOL2 plasmid was constructed. The VrCOL2 CDS was amplified from the cDNA of the sequenced mungbean variety VC1973A using primers with Xhol and $X$ bal digestion site sequences. The resulting PCR fragment was digested by the restriction endonucleases $X$ hol and $X b a l$ to generate cohesive ends. The pRTL2 vector was digested with $X$ hol and $X b a l$ to generate a linearized plasmid. Then the $\operatorname{VrCOL} 2$ and pRTL2 fragments were ligated using T4 DNA ligase (Promega). The constructed plasmid was verified by sequencing. It was then introduced into $A$. thaliana using the floral dip method [69], and successful transformation was confirmed by PCR. In addition, the VrCOL2 overexpression construct was transformed into VC1973A to obtain transgenic root hairs using the method as described by Kereszt et al. [70]. Three-week old transgenic root hairs grown under LD and SD conditions were collected for gene expression analysis, respectively. Each sample was analyzed using three independent root hairs. All primers are listed in Additional file 9.

\section{RNA extraction and transcription analysis}

RNA isolation and quantitative real-time PCR (qRT-PCR) analysis were carried out as described in Li et al. [49]. Gene expression levels were normalized to an Actin gene from mungbean (Vradi03g00210). Each sample was analyzed using three biological replicates. All primers are listed in Additional file 9.

\section{Declarations}

\section{Ethics approval and consent to participate}




\section{Consent to publish}

Not applicable.

\section{Availability of data and materials}

The datasets generated and analyzed during the current study are available from the corresponding author on reasonable request.

\section{Competing interests}

The authors declare that they have no competing interests.

\section{Funding}

This research was funded by the National Natural Science Foundation of China (grant 31971898), the Qingdao Agricultural University Scientific Research Foundation (grant 6631119010, 6651118005), and the National Key R \& D Project (grant 2016YFD0100304, 2016YFD0101005). The funders have no role in the design of the study and collection, analysis, and interpretation of data and in writing the manuscript.

\section{Authors' Contributions}

SL conceived and designed the research. CL, QZ, HZ and CC conducted the experiments and analyzed the data. SL and $\mathrm{HZ}$ wrote the manuscript. All authors read and approved the manuscript.

\section{Acknowledgements}

We thank Suk-Ha Lee at Seoul National University, Seoul, Korea, for supplying mungbean VC1973A seeds.

\section{Abbreviations}

BBX, B-box; CCT, CONSTANS, CONSTANS-LIKE and TIMING OF CAB1; LD, Long day; SD, Short day; DNA, Deoxyribonucleic acid; RNA, Ribonucleic acid; CDS, Coding domain sequence; UTR, Untranslated Regions; AA, Amino acid; CO, CONSTANS; FT, FLOWERING LOCUS T ; COL, CO-like; GSDS, Gene Structure Display Server program; $\mathrm{Vr}$,Vigna radiate; $\mathrm{Gm}$, Glycine max; pl, Isoelectric point; MW, Molecular weight; OsHd1, 
Heading date 1; OsHd3a, Heading date 3a; NCBI, National Center for Biotechnology Information; qRT-PCR, quantitative real-time PCR.

\section{References}

1. Wickland DP, Hanzawa Y. The FLOWERING LOCUS T/TERMINAL FLOWER 1 gene family: functional evolution and molecular mechanisms. Mol Plant. 2015; 8(7):983-997.

2. Beinecke FA, Grundmann L, Wiedmann DR, Schmidt FJ, Caesar AS, Zimmermann M, Lahme M, Twyman RM, Prufer D, Noll GA. The FT/FD-dependent initiation of flowering under long-day conditions in the day-neutral species Nicotiana tabacum originates from the facultative short-day ancestor Nicotiana tomentosiformis. Plant J. 2018; 96(2):329-342.

3. Eom H, Park SJ, Kim MK, Kim H, Kang H, Lee I. TAF15b, involved in the autonomous pathway for flowering, represses transcription of FLOWERING LOCUS C. Plant J. 2018; 93(1):79-91.

4. Xu S, Chong K. Remembering winter through vernalisation. Nat Plants. 2018; 4(12):997-1009.

5. Boss PK, Bastow RM, Mylne JS, Dean C. Multiple pathways in the decision to flower: enabling, promoting, and resetting. Plant Cell. 2004; 16 Suppl:S18-31.

6. Jack T. Molecular and genetic mechanisms of floral control. Plant Cell. 2004; 16 Suppl:S1-17.

7. Baurle I, Dean C. The timing of developmental transitions in plants. Cell. 2006; 125(4):655-664.

8. Ronald J, Davis SJ. Focusing on the nuclear and subnuclear dynamics of light and circadian signaling. Plant Cell Environ. 2019; 42:2871-2884.

9. Taylor CM, Kamphuis LG, Zhang W, Garg G, Berger JD, Mousavi-Derazmahalleh M, Bayer PE, Edwards $D$, Singh KB, Cowling WA, et al. INDEL variation in the regulatory region of the major flowering time gene $L a n F T c 1$ is associated with vernalization response and flowering time in narrow-leafed lupin (Lupinus angustifolius L.) . Plant Cell Environ. 2019; 42:174-187.

10. Zhang W, Yuan J, Cheng T, Tang MJ, Sun K, Song SL, Xu FJ, Dai CC. Flowering-mediated root-fungus symbiosis loss is related to jasmonate-dependent root soluble sugar deprivation. Plant Cell Environ. 2019; 42(12):3208-3226.

11. Gangappa SN, Botto JF. The BBX family of plant transcription factors. Trends Plant Sci. 2014; 19(7):460-470.

12. del-Olmo I, Poza-Viejo L, Piñeiro M, Jarillo JA, Crevillén P. High ambient temperature leads to reduced $F$ Texpression and delayed flowering in Brassica rapavia a mechanism associated with H2AZ dynamics. Plant J. 2019; 100:343-356.

13. Jin H, Tang X, Xing M, Zhu H, Sui J, Cai CM, Li S. Molecular and transcriptional characterization of phosphatidyl ethanolamine-binding proteins in wild peanuts Arachis duranensis and Arachis ipaensis. BMC Plant Biol. 2019; 19:484.

14. Jing $Y, G u o$ Q, Zha P, Lin R. The chromatin-remodeling factor PICKLE interacts with CONSTANS to promote flowering in Arabidopsis. Plant Cell Environ. 2019; 42:2495-2507. 
15. Lee C, Kim S, Jin S, Susila H, Youn G, Nasim Z, Alavilli H, Chung K, Yoo SJ, Ahn JH. Genetic interactions reveal the antagonistic roles of FT/TSFand TFL 1 in the determination of inflorescence meristem identity in Arabidopsis. Plant J. 2019; 99:452-464.

16. Nam J, dePamphilis CW, Ma H, Nei M. Antiquity and evolution of the MADS-box gene family controlling flower development in plants. Mol Biol Evol. 2019; 20:1435-1447.

17. Ning Y, Chen Q, Lin R, Li Y, Li L, Chen S, He X. The HDA19 histone deacetylase complex is involved in the regulation of flowering time in a photoperiod-dependent manner. Plant J. 2019; 98:448-464.

18. Parenicova L, de Folter S, Kieffer M, Horner DS, Favalli C, Busscher J, Cook HE, Ingram RM, Kater MM, Davies $B$, et al. Molecular and phylogenetic analyses of the complete MADS-box transcription factor family in Arabidopsis: new openings to the MADS world. Plant Cell. 2019; 15:1538-1551.

19. Jin H, Xing M, Cai C, Li S. B-box proteins in Arachis duranensis: genome-wide characterization and expression profiles analysis. Agronomy. 2020; 10:23.

20. Khanna R, Kronmiller B, Maszle DR, Coupland G, Holm M, Mizuno T, Wu SH. The ArabidopsisB-box zinc finger family. Plant Cell. 2019; 21(11):3416-3420.

21. Robson F, Costa MMR, Hepworth SR, Vizir I, Pineiro M, Reeves PH, Putterill J, Coupland G. Functional importance of conserved domains in the flowering-time gene CONSTANS demonstrated by analysis of mutant alleles and transgenic plants. Plant J. 2001; 28:619-631.

22. Yan H, Marquardt K, Indorf M, Jutt D, Kircher S, Neuhaus G, Rodríguez-Franco M. Nuclear localization and interaction with COP1 are required for STO/BBX24 function during photomorphogenesis. Plant Physiol. 2011; 156:1772-1782.

23. Luo X, Gao Z, Wang Y, Chen Z, Zhang W, Huang J, Yu H, He Y. The NUCLEAR FACTOR-CONSTANS complex antagonizes polycomb repression to de-repress FLOWERING LOCUS T expression in response to inductive long days in Arabidopsis. Plant J. 2018; 95:17-29.

24. Luccioni L, Krzymuski M, Sánchez-Lamas M, Karayekov E, Cerdán PD, Casal JJ. CONSTANS delays Arabidopsis flowering under short days. Plant J. 2019; 97:923-932.

25. Serrano-Bueno G, Said FE, de los Reyes P, Lucas-Reina El, Ortiz-Marchena MI, Romero JM, Valverde F. CONSTANS-FKBP12 interaction contributes to modulate photoperiodic flowering in Arabidopsis. Plant J. 2020; 101: 1287-1302.

26. Putterill J, Robson F, Lee K, Coupland G. Chromosome walking with YAC clones in Arabidopsis: isolation of $1700 \mathrm{~kb}$ of contiguous DNA on chromosome 5, including a $300 \mathrm{~kb}$ region containing the flowering-time gene CO. Molec Gen Genet. 1993; 239:145-157.

27. Putterill J, Robson F, Lee K, Simon R, Coupland G. The CONSTANS gene of Arabidopsis promotes flowering and encodes a protein showing similarities to zinc finger transcription factors. Cell. 1995; 80(6):847-857.

28. Andres F, Coupland G. The genetic basis of flowering responses to seasonal cues. Nat Rev Genet. 2012; 13(9):627-639.

29. Song YH, Ito S, Imaizumi T. Flowering time regulation: photoperiod-and temperature-sensing in leaves. Trends Plant Sci. 2013; 18(10):575-583. 
30. Imaizumi T, Schultz TF, Harmon FG, Ho LA, Kay SA. FKF1 F-Box protein mediates cyclic degradation of a repressor of CONSTANS in Arabidopsis. Science. 2005; 309(5732):293-297.

31. Sawa M, Nusinow DA, Kay SA, Imaizumi T. FKF1 and GIGANTEA complex formation is required for day-length measurement in Arabidopsis. Science. 2007; 318(5848):261-265.

32. Yano M, Katayose Y, Ashikari M, Yamanouchi U, Monna L, Fuse T, Baba T, Yamamoto K, Umehara Y, Nagamura $\mathrm{Y}$, et al. $H d 1$, a major photoperiod sensitivity quantitative trait locus in rice, is closely related to the Arabidopsis flowering time gene CONSTANS. Plant Cell. 2000; 12(12):2473-2483.

33. Komiya R, Ikegami A, Tamaki S, Yokoi S, Shimamoto K. Hd3a and RFT1 are essential for flowering in rice. Development. 2008; 135(4):767-774.

34. Komiya R, Yokoi S, Shimamoto K. A gene network for long-day flowering activates RFT1 encoding a mobile flowering signal in rice. Development. 2009; 136(20):3443-3450.

35. Wu F, Price BW, Haider W, Seufferheld G, Nelson R, Hanzawa Y. Functional and evolutionary characterization of the CONSTANS gene family in short-day photoperiodic flowering in soybean. PLoS One. 2014; 9(1):e85754.

36. Keatinge JDH, Easdown WJ, Yang RY, Chadha ML, Shanmugasundaram S. Overcoming chronic malnutrition in a future warming world: the key importance of mungbean and vegetable soybean. Euphytica. 2011; 180:129-141.

37. Fuller DQ. Contrasting patterns in crop domestication and domestication rates: recent archaeobotanical insights from the old world. Ann Bot. 2007; 100:903-924.

38. Imrie BC. Mung bean, in The New Rural Industries: A Handbook for Farmers and Investors, ed. K. Hyde (Canberra: Rural Industries Research and Development Corporation), 1996; pp. 355-360.

39. Kim SK, Nair RM, Lee J, Lee SH. Genomic resources in mungbean for future breeding programs. Front Plant Sci. 2015; 6:626.

40. Vas Aggarwal D, Poehlman J. Effects of photoperiod and temperature on flowering in mungbean (Vigna radiata(L.) WILCZEK). Euphytica. 1977; 26: 207-219.

41. Kumari P, Verma SK. Genotypic differences in flower production, shedding and yield in mungbean. J Agric Sci. 1983; 99:219-223.

42. Mondal MMA, Fakir MSA, Juraimi AS, Hakim MA, Islam MM, Shamsuddoha ATM. Effects of flowering behavior and pod maturity synchrony on yield of mungbean [Vigna radiata (L.) Wilczek]. Aust J Crop Sci. 2011; 5:945-953.

43. Isobe $K$, Kokubun M, Tsuboki Y. Effects of soybean raceme-order on pod set and seed growth in three cultivars. Jpn J Crop Sci. 1995; 64:281-287.

44. Kuroda T, Saitoh K, Mahmood T, Yanagawa K. Differences in flowering habit between determinate and indeterminate types of soybean. Plant Prod Sci. 1998; 1:18-24.

45. Kang YJ, Kim SK, Kim MY, Lestari P, Kim KH, Ha BK, Jun TH, Hwang WJ, Lee T, Lee J, et al. Genome sequence of mungbean and insights into evolution within Vigna species. Nat Commun. 2014; 5:5443. 
46. Hu T, Wei Q, Wang W, Hu H, Mao W, Zhu Q, Bao C. Genome-wide identification and characterization of CONSTANS-like gene family in radish (Raphanus sativus). PLoS ONE.2018; 13(9):e0204137.

47. Wu W, Zheng XM, Chen D, Zhang Y, Ma W, Zhang H, Sun I, Yang Z, Zhao C, Zhan X, Shen X, Yu P, Fu Y, Zhu S, Cao L, Cheng S. OsCOL16, encoding a CONSTANS-like protein, represses flowering by upregulating Ghd7 expression in rice. Plant Sci. 2017; 260: 60-69.

48. Hu B, Jin J, Guo A, Zhang H, Luo J, Gao G. GSDS 2.0: an upgraded gene feature visualization server. Bioinformatics. 2015; 31(8):1296-1297.

49. Li S, Wang R, Jin H, Ding Y, Cai C. Molecular characterization and expression profile analysis of heat shock transcription factors in mungbean. Front Genet. 2019; 9:736.

50. Lescot M, Dehais P, Thijs G, Marchal K, Moreau Y, Van de Peer Y, Rouze P, Rombauts S. PlantCARE, a database of plant cis-acting regulatory elements and a portal to tools for in silico analysis of promoter sequences. Nucleic Acids Res. 2002; 30:325-327.

51. Kondrashov FA, Rogozin IB, Wolf YI, Koonin EV. Selection in the evolution of gene duplications. Genome Biol. 2002; 3:RESEARCH0008.

52. Wang Z, Zhou Z, Liu Y, Liu T, Li Q, Ji Y, Li C, Fang C, Wang M, Wu M, et al. Functional evolution of phosphatidyl ethanolamine binding proteins in soybean and Arabidopsis. Plant Cell. 2015; 27:323336.

53. Suárez-López P, Wheatley K, Robson F, Onouchi H, Valverde F, Coupland G. CONSTANSmediates between the circadian clock and the control of flowering in Arabidopsis. Nature. 2001; 410:11161120.

54. Wong ACS, Hecht VFG, Picard K, Diwadkar P, Laurie RE, Wen J, Mysore K, Macknight RC, Weller JL. Isolation and functional analysis of CONSTANS-LIKEgenes suggests that a central role for CONSTANS in flowering time control is not evolutionarily conserved in Medicago truncatula. Front Plant Sci. 2014; 5:486.

55. Initiative AG. Analysis of the genome sequence of the flowering plant Arabidopsis thaliana. Nature. 2000; 408:796-815.

56. Schmutz J, Cannon S, Schlueter J, Ma J, Mitros T, Nelson W, Hyten DL, Song Q, Thelen JJ, Cheng J, et al. Genome sequence of the palaeopolyploid soybean. Nature. 2010; 463:178-183.

57. Young N, Debellé F, Oldroyd G, Geurts R, Cannon SB, Udvardi MK, Benedito VA, Mayer KF, Gouzy J, Schoof $\mathrm{H}$, et al. The Medicagogenome provides insight into the evolution of rhizobial symbioses.Nature. 2011; 480:520-524.

58. Shim JS, Imaizumi T. Circadian clock and photoperiodic response in Arabidopsis. from seasonal flowering to redox homeostasis. Biochemistry. 2015; 54:157-170.

59. Shim JS, Kubota A, Imaizumi T. Circadian clock and photoperiodic flowering in Arabidopsis: CONSTANS is a hub for signal integration. Plant Physiol. 2017; 173 (1):5-15.

60. Song YH, Shim JS, Kinmonth-Schultz HA, Imaizumi T. Photoperiodic flowering: time measurement mechanisms in leaves. Annu Rev Plant Biol. 2015; 66:441-464. 
61. El-Gebali S, Mistry J, Bateman A, Eddy S, Luciani A, Potter S, Qureshi M, Richardson L, SalazarG, Smart A, et al. The Pfam protein families database in 2019.Nucleic Acids Res. 2019; 47:427-432.

62. Finn R, Attwood T, Babbitt P, Bateman A, Bork P, Bridge A, Chang H, Dosztányi Z, El-Gebali S, Fraser M, et al. InterPro in 2017-beyond protein family and domain annotations. Nucleic Acids Res. 2017; 45:190-199.

63. Oliver T, Schmidt B, Nathan D, Clemens R, Maskell D. Using reconfigurable hardware to accelerate multiple sequence alignment with clustalW. Bioinformatics. 2005; 21:3431-3432.

64. Kumar S, Stecher G, Tamura K. MEGA7: Molecular evolutionary genetics analysis version 7.0 for bigger datasets. Mol Biol Evol. 2016; 33:1870-1874.

65. Fischer S, Brunk BP, Chen F, Gao X, Harb OS, lodice JB, Shanmugam D, Roos DS, Stoeckert CJ. Using OrthoMCL to assign proteins to OrthoMCL-DB groups or to cluster proteomes into new ortholog groups. Curr Protoc Bioinformatics. 2011; 35:1-19.

66. Krzywinski M, Schein J, Birol I, Connors J, Gascoyne R, Horsman D, Jones SJ, Marra MA. Circos: An information aesthetic for comparative genomics. Genome Res. 2009; 19:1639-1645.

67. Crooks GE, Hon G, Chandonia JM, Brenner SE. WebLogo: a sequence logo generator. Genome Res. 2004; 14(6):1188-1190.

68. Bailey T, Boden M, Buske F, Frith M, Grant C, Clementi L, Ren J, Li W, Noble W. MEME suite: Tools for motif discovery and searching. Nucleic Acids Res. 2009; 37:W202-W208.

69. Bent A. Arabidopsis thaliana floral dip transformation method. Methods Mol Biol. 2006; 343:87-103.

70. Kereszt A, Li D, Indrasumunar A, Nguyen C, Nontachaiyapoom S, Kinkema M, Gresshoff P. Agrobacterium rhizogenes-mediated transformation of soybean to study root biology. Nat Protoc. 2007; 2: 948-952.

\section{Supplementary Files}

Additional file 1: Sequence alignments of conserved B-Box1, B-Box2 and CCT domains of VrCOL proteins. Additional file 2: Sequence logos of 17 distinct motifs in VrCOL proteins.

Additional file 3: Cis-acting elements identified in each VrCOL promoter regions.

Additional file 4: Relative expression levels of VrCOL duplicated genes in different tissues. The expression level of VrCOL 1 in flowers was set as 1, and the others were adjusted accordingly. *** and ** are significantly different at $\mathrm{P}<0.001$ and $\mathrm{P}<0.01$, respectively, compared with the expression levels in its duplicated gene.

Additional file 5: The rosette leaf numbers of empty vector transgenic lines and wild-type plants grown under LD and SD conditions. 
Additional file 6: Expression analysis of VrCOL2 in VrCOL2 transgenic lines and wild-type A. thaliana measured by qRT-PCR. The leaves of transgenic and wild-type plants grown under LD conditions were sampled $5 \mathrm{~h}$ after lights-on for qRT-PCR analysis. Gene expression levels were normalized to an Actin gene from $A$. thaliana. ND, not detected.

Additional file 7: Expression levels of two mungbean $F T$ homologous genes in VrCOL2 transgenic root hairs. (a) The positions of CORE (TGTG(N2-3)ATG motif) cis-acting elements in the promoter regions of mungbean FT homologous genes XP_014496932, XP_014497364. The red boxes in the promoter regions indicate CORE cis-acting elements. (b) The expression levels of XP_014496932, XP_014497364 in VrCOL2 transgenic root hairs under LD and SD conditions. The empty vector was transformed as a control. Tissues were sampled 5 hours after lights-on (ZT 5). The gene expression in each control plants was set as 1 and those in other samples were adjusted accordingly. ${ }^{\star} * \star P<0.001$.

Additional file 8: Expression levels of VrCOL2 in leaves at three growth stages, before flowering (S1), during flowering (S2), and after flowering (S3).

Additional file 9: Primers used in this study.

\section{Tables}

Table 1. $\operatorname{VrCOL}$ genes identified in mungbean genome

\begin{tabular}{|c|c|c|c|c|c|c|c|c|c|}
\hline Gene ID & $\begin{array}{c}\text { Genomic } \\
\text { length/bp }\end{array}$ & $\mathrm{CDS} / \mathrm{bp}$ & $\begin{array}{c}\text { No. of } \\
\text { AA }\end{array}$ & pI & $\begin{array}{c}\text { Mol.Wt } \\
\text { /Da }\end{array}$ & GC\% & $\mathrm{Chr}$ & Strand & Gene names \\
\hline XP_014498167 & 2481 & 1074 & 357 & 5.82 & 39253.5 & 42.93 & 4 & - & $\operatorname{VrCOL1}$ \\
\hline XP_014509740 & 2195 & 1071 & 356 & 5.27 & 39685.26 & 40.68 & 1 & - & $\operatorname{VrCOL2}$ \\
\hline XP_014502470 & 1506 & 933 & 310 & 7.01 & 33756.82 & 50.13 & 5 & - & $\operatorname{VrCOL3}$ \\
\hline XP_014510275 & 1778 & 1119 & 372 & 6.11 & 40396.31 & 50.39 & 7 & - & $\operatorname{VrCOL4}$ \\
\hline XP_014511026 & 3495 & 1239 & 412 & 4.95 & 46524.78 & 36.89 & 1 & + & $\operatorname{VrCOL5}$ \\
\hline XP_014505914 & 2175 & 1110 & 369 & 5.64 & 41395.12 & 40.37 & 7 & + & $\operatorname{VrCOL6}$ \\
\hline XP_014512653 & 2060 & 1254 & 417 & 5.28 & 46964.34 & 43.83 & 8 & - & $\operatorname{VrCOL} 7 a$ \\
\hline XP_014523547 & 1960 & 1113 & 370 & 9.22 & 42031.83 & 40.10 & $\mathrm{~N} / \mathrm{A}$ & + & $\operatorname{VrCOL7b}$ \\
\hline XP_014505158 & 8864 & 1236 & 411 & 5.21 & 45045.34 & 34.64 & 6 & + & $\operatorname{VrCOL8}$ \\
\hline XP_014523701 & 14007 & 1230 & 409 & 4.86 & 44487.59 & 40.33 & 5 & + & VrCOL9 \\
\hline XP_022637309 & 4494 & 1329 & 442 & 6.47 & 48806.9 & 42.28 & 5 & + & $\operatorname{VrCOL10}$ \\
\hline XP_014496130 & 4536 & 1035 & 344 & 6.47 & 38345.14 & 45.16 & 3 & - & $\operatorname{VrCOL11}$ \\
\hline XP_022641108 & 3819 & 954 & 317 & 6.82 & 35966.39 & 40.02 & 8 & - & $\operatorname{VrCOL12}$ \\
\hline XP_014497035 & 3750 & 1119 & 372 & 7.00 & 41848.9 & 39.46 & 4 & + & $\operatorname{VrCOL13}$ \\
\hline
\end{tabular}

Chr, chromosome number; AA, amino acid; Mol.Wt, molecular weight; pI, isoelectric point; N/A, not applicable.

Table 2. Numbers and types of cis-acting elements in each $\operatorname{VrCOL}$ promoter region 


\begin{tabular}{|c|c|c|c|c|c|c|c|}
\hline $\begin{array}{l}\text { Gene } \\
\text { name }\end{array}$ & $\begin{array}{c}\text { Development } \\
\text { related } \\
\text { elements }\end{array}$ & $\begin{array}{c}\text { Environmental } \\
\text { stress related } \\
\text { elements }\end{array}$ & $\begin{array}{l}\text { Hormone- } \\
\text { responsive } \\
\text { elements }\end{array}$ & $\begin{array}{l}\text { Light- } \\
\text { responsive } \\
\text { elements }\end{array}$ & $\begin{array}{l}\text { Promoter } \\
\text { related } \\
\text { elements }\end{array}$ & $\begin{array}{l}\text { Site-binding } \\
\text { related } \\
\text { elements }\end{array}$ & Others \\
\hline $\operatorname{VrCOL1}$ & 0 & 3 & 4 & 11 & 2 & 0 & 18 \\
\hline $\operatorname{VrCOL2}$ & 1 & 3 & 4 & 6 & 2 & 1 & 18 \\
\hline $\operatorname{VrCOL3}$ & 2 & 1 & 4 & 8 & 2 & 0 & 19 \\
\hline $\operatorname{VrCOL4}$ & 1 & 0 & 4 & 11 & 2 & 2 & 17 \\
\hline $\operatorname{VrCOL5}$ & 1 & 3 & 3 & 6 & 2 & 0 & 17 \\
\hline $\operatorname{VrCOL} 6$ & 1 & 0 & 4 & 11 & 2 & 2 & 17 \\
\hline $\operatorname{VrCOL} 7 a$ & 1 & 1 & 5 & 8 & 2 & 2 & 17 \\
\hline $\operatorname{VrCOL7b}$ & 0 & 0 & 4 & 7 & 2 & 0 & 14 \\
\hline $\operatorname{VrCOL8}$ & 1 & 9 & 4 & 8 & 2 & 0 & 13 \\
\hline $\operatorname{VrCOL9}$ & 0 & 1 & 5 & 7 & 2 & 0 & 14 \\
\hline $\operatorname{VrCOL} 10$ & 1 & 2 & 4 & 6 & 2 & 1 & 14 \\
\hline $\operatorname{VrCOL11}$ & 0 & 2 & 5 & 6 & 3 & 0 & 20 \\
\hline $\operatorname{VrCOL12}$ & 0 & 3 & 4 & 7 & 2 & 1 & 16 \\
\hline $\operatorname{VrCOL13}$ & 4 & 1 & 4 & 6 & 2 & 2 & 15 \\
\hline
\end{tabular}

Figures 


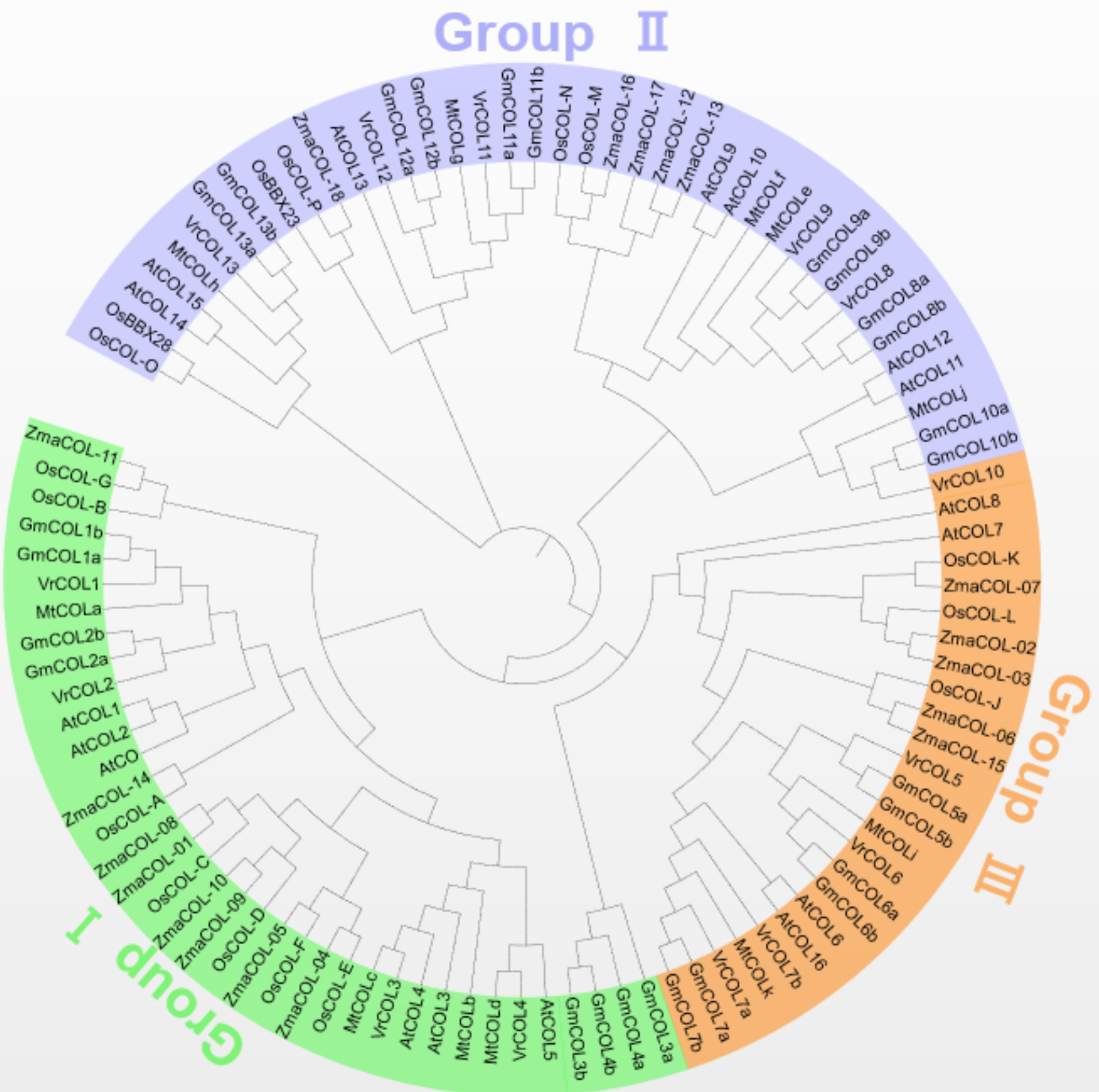

Figure 1

Phylogenetic analysis of the evolutionary relationships among $\mathrm{VrCOL}$ proteins and $\mathrm{COL}$ proteins from other species. The amino acid sequences of COL proteins from A. thaliana, soybean, Medicago, mungbean, rice and maize were used to construct the phylogenetic tree in MEGA7.0. Different groups of $\operatorname{VrCOL}$ genes are indicated. 

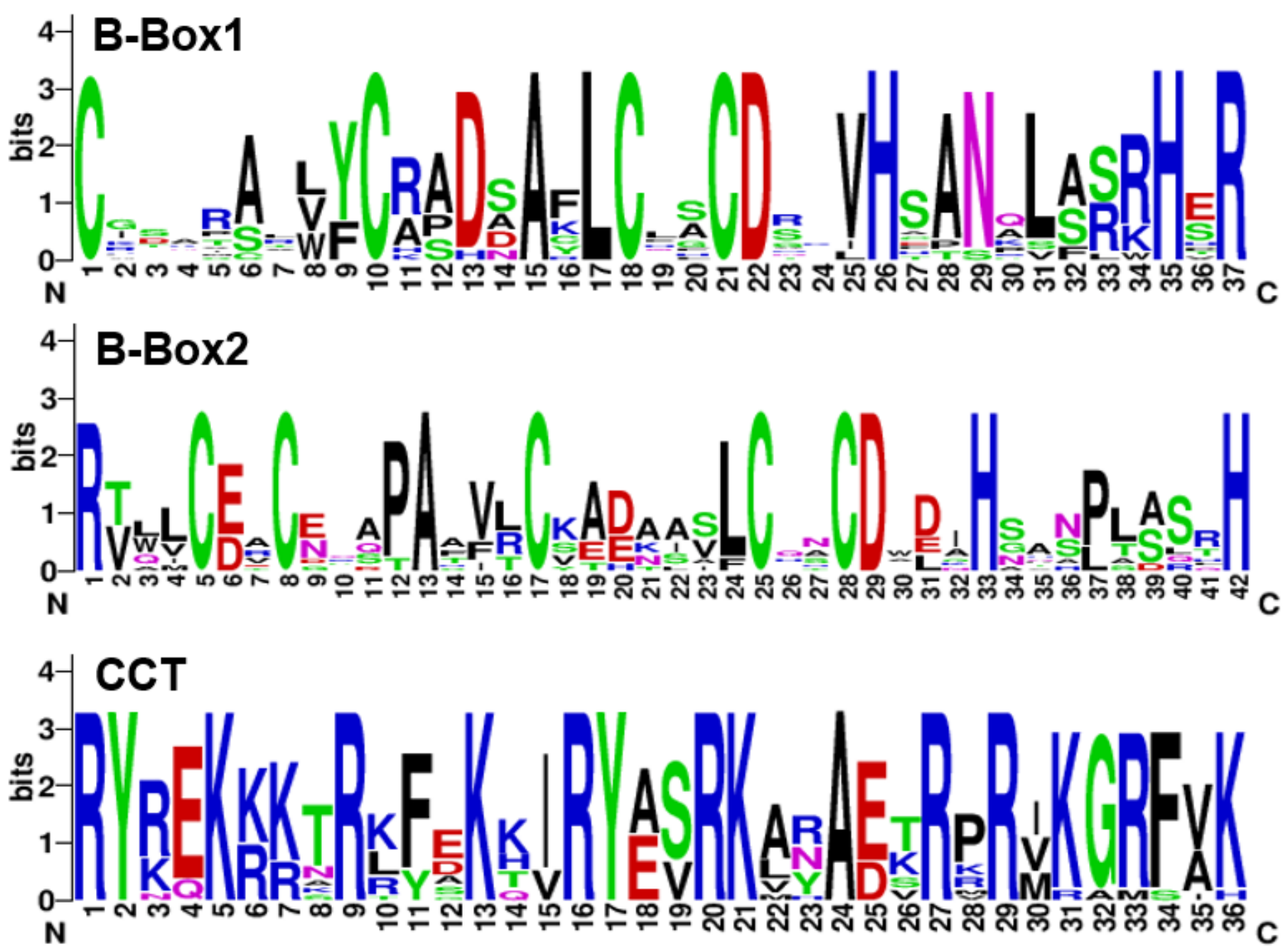

Figure 2

Sequence logos of the BBX1, BBX2 and CCT domains of $\mathrm{VrCOL}$ proteins. The conserved domains were analyzed using the WebLogo platform. 


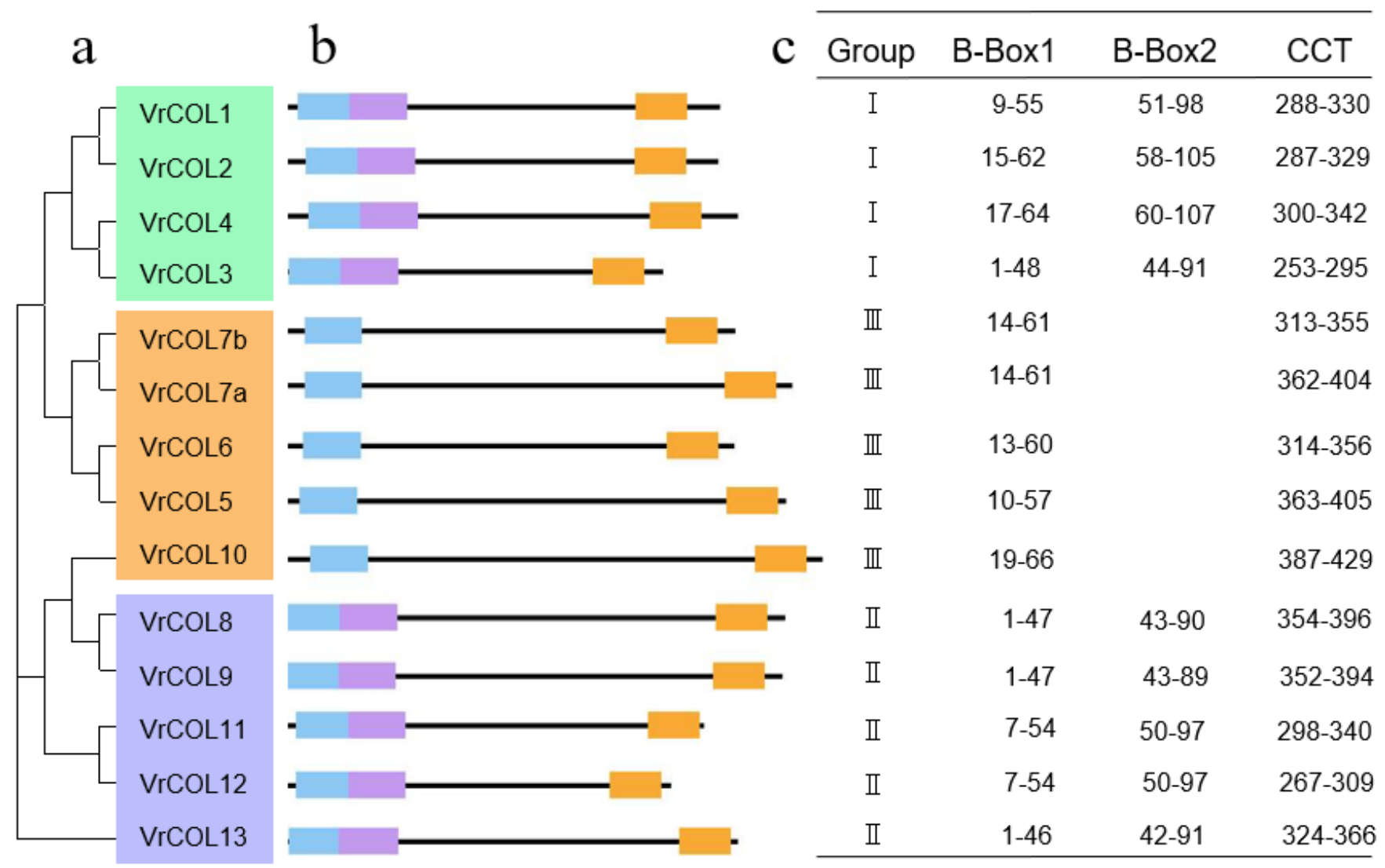

$\begin{array}{ccccccccc}1 & 1 & 1 & 1 & 1 & 1 & 1 & 1 & 1 \\ 1 & 50 & 100 & 150 & 200 & 250 & 300 & 350 & 400\end{array}$

\section{Figure 3}

Phylogenetic relationship and conserved domain analyses of the $\mathrm{VrCOL}$ proteins. (a) $\mathrm{VrCOL}$ protein sequences were used to construct a phylogenetic tree. (b) The positions of conserved BBX1, BBX2 and CCT domains in the $\mathrm{VrCOL}$ proteins. The blue, purple and orange boxes indicate the BBX1, BBX2 and CCT domains, respectively. (c) Classifications and conserved domain positions of the $\mathrm{VrCOL}$ proteins. 
a

\section{Gene Name Gene structure}

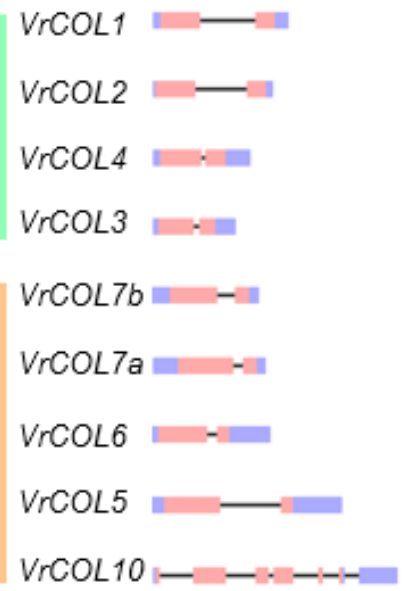

$\operatorname{VrCOL8}=\mathrm{H}-\mathrm{-}$

$\operatorname{VrCOL9}$

$\operatorname{VrCOL11}-\cdots$

$\operatorname{VrCOL12~}$

$\operatorname{VrCOL13}$ $\mathrm{b}$

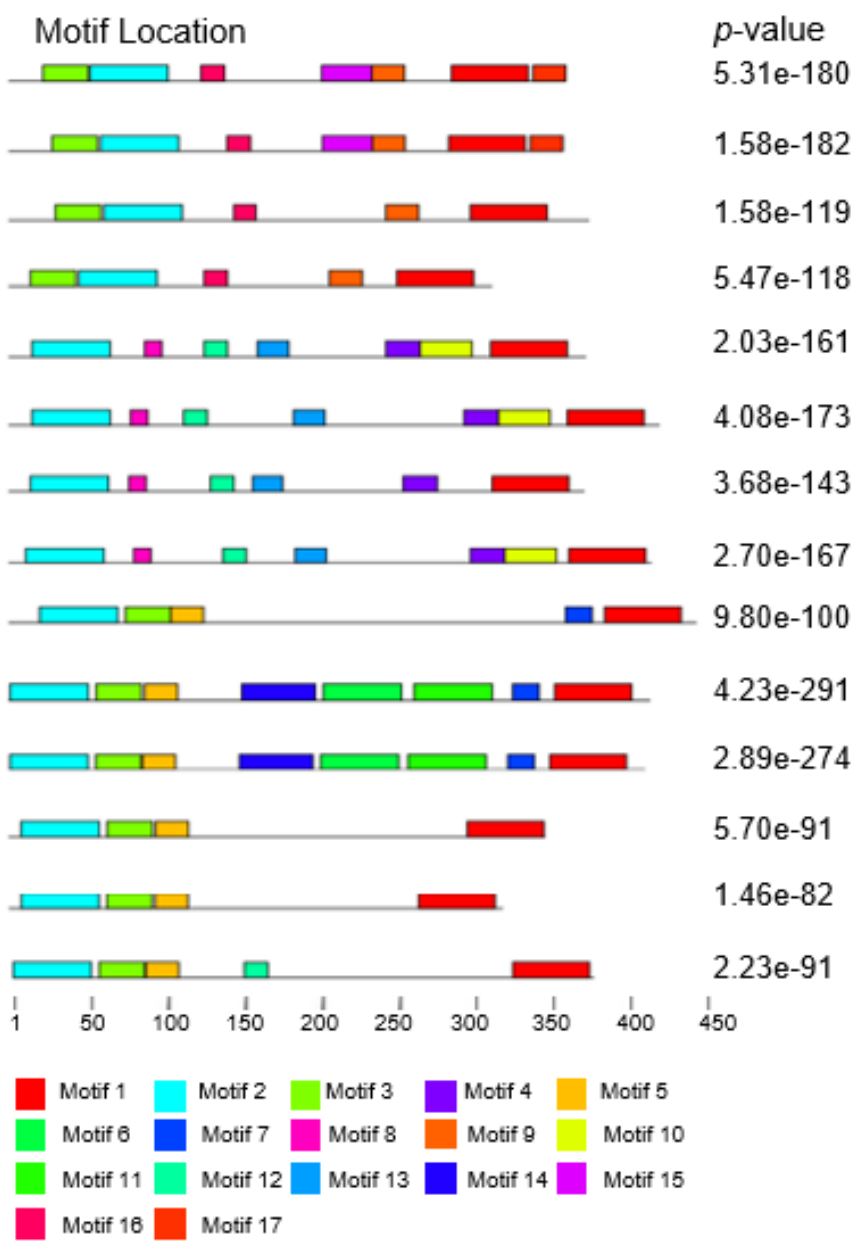

\section{Figure 4}

Gene structures and conserved motifs of the VrCOL proteins. (a) Exon-intron organizations of VrCOL genes. The blue boxes, pink boxes and black lines indicate UTRs, exons and introns, respectively. (b) Conserved motifs of the $\mathrm{VrCOL}$ proteins. Different motifs are indicated by different colored boxes. 

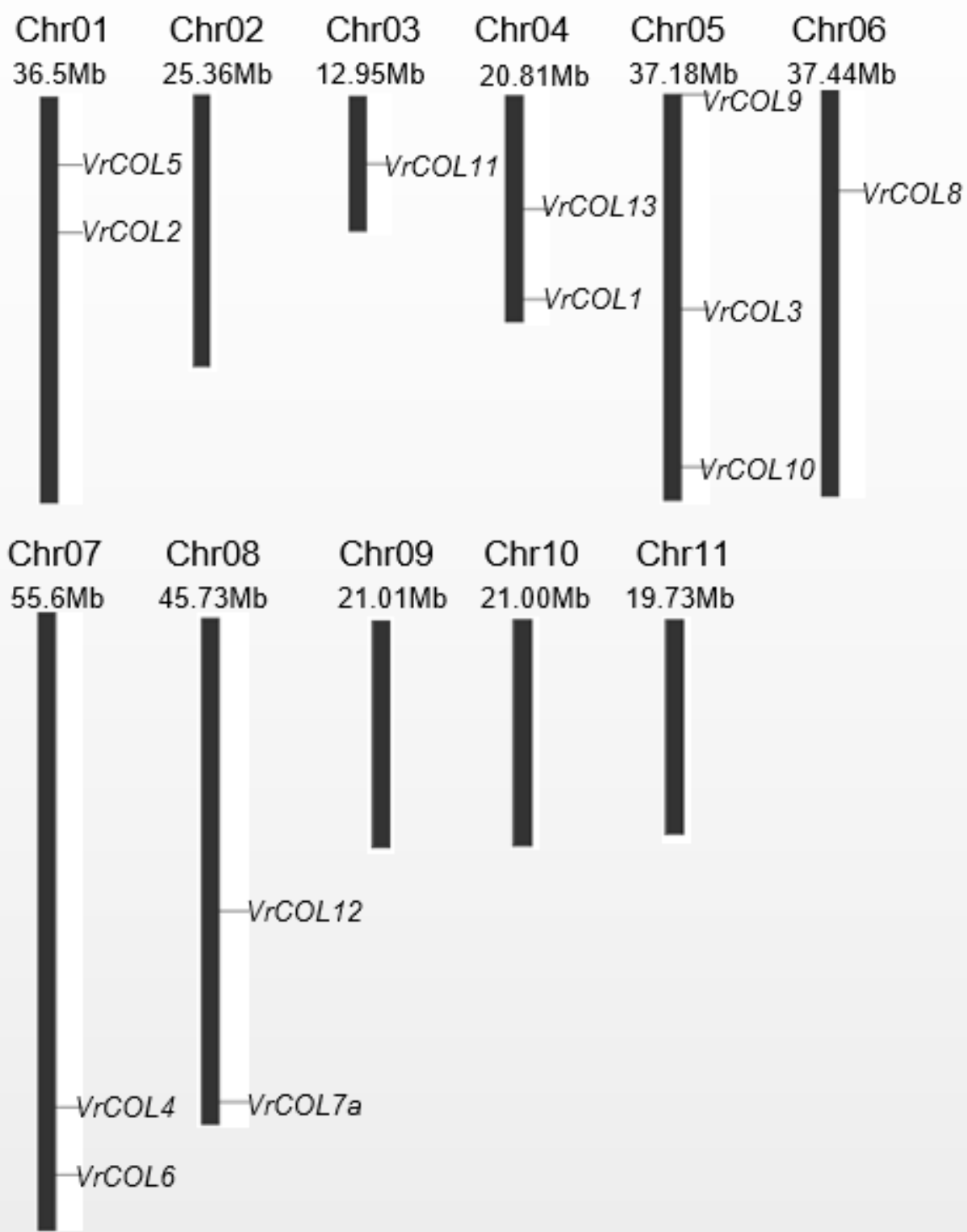

Figure 5

Chromosomal locations of the $\mathrm{VrCOL}$ genes. Chromosome number and length are indicated. 


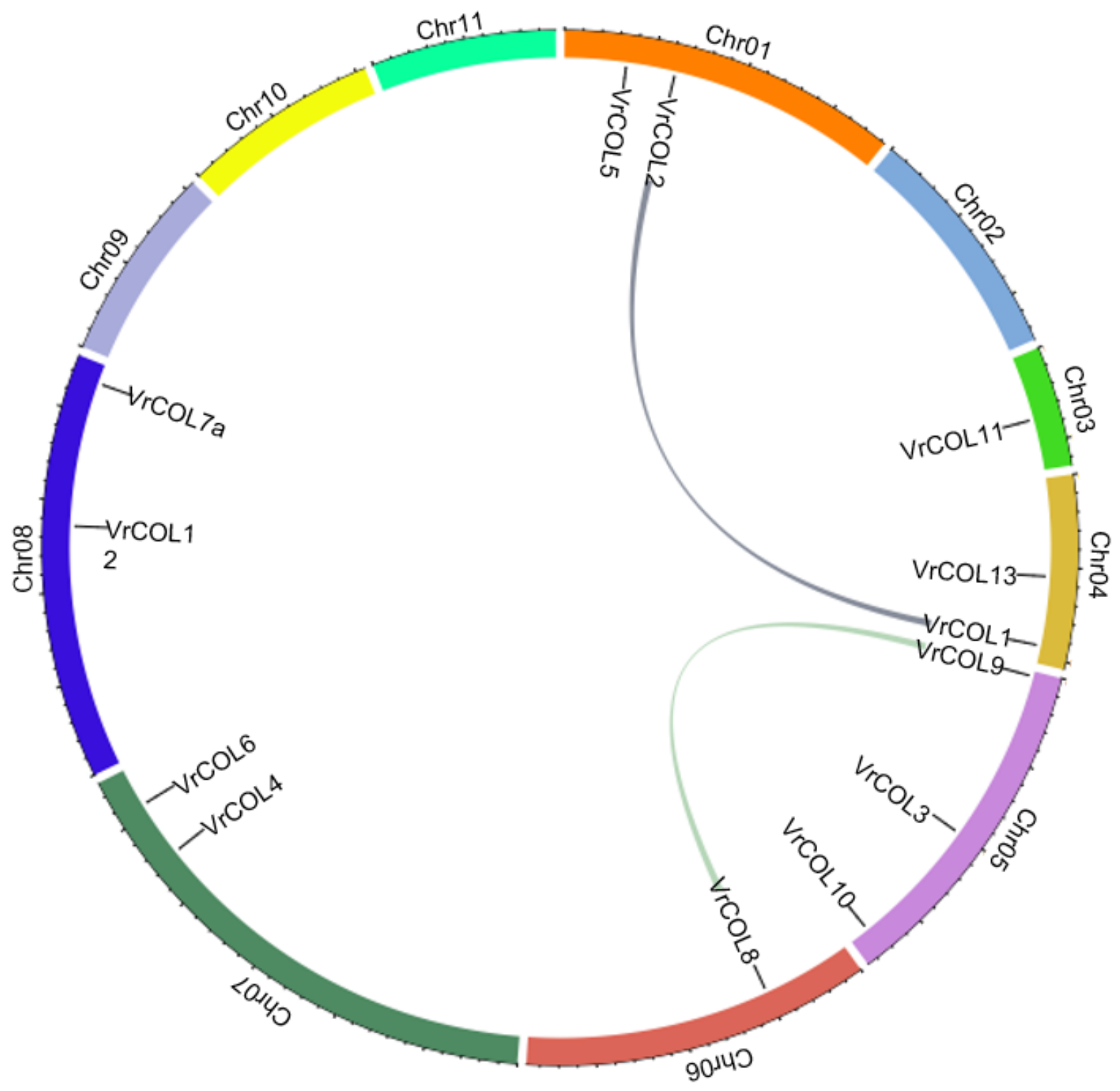

Figure 6

Duplication analysis of $\mathrm{VrCOL}$ proteins. The duplicated gene pairs are connected by lines. 

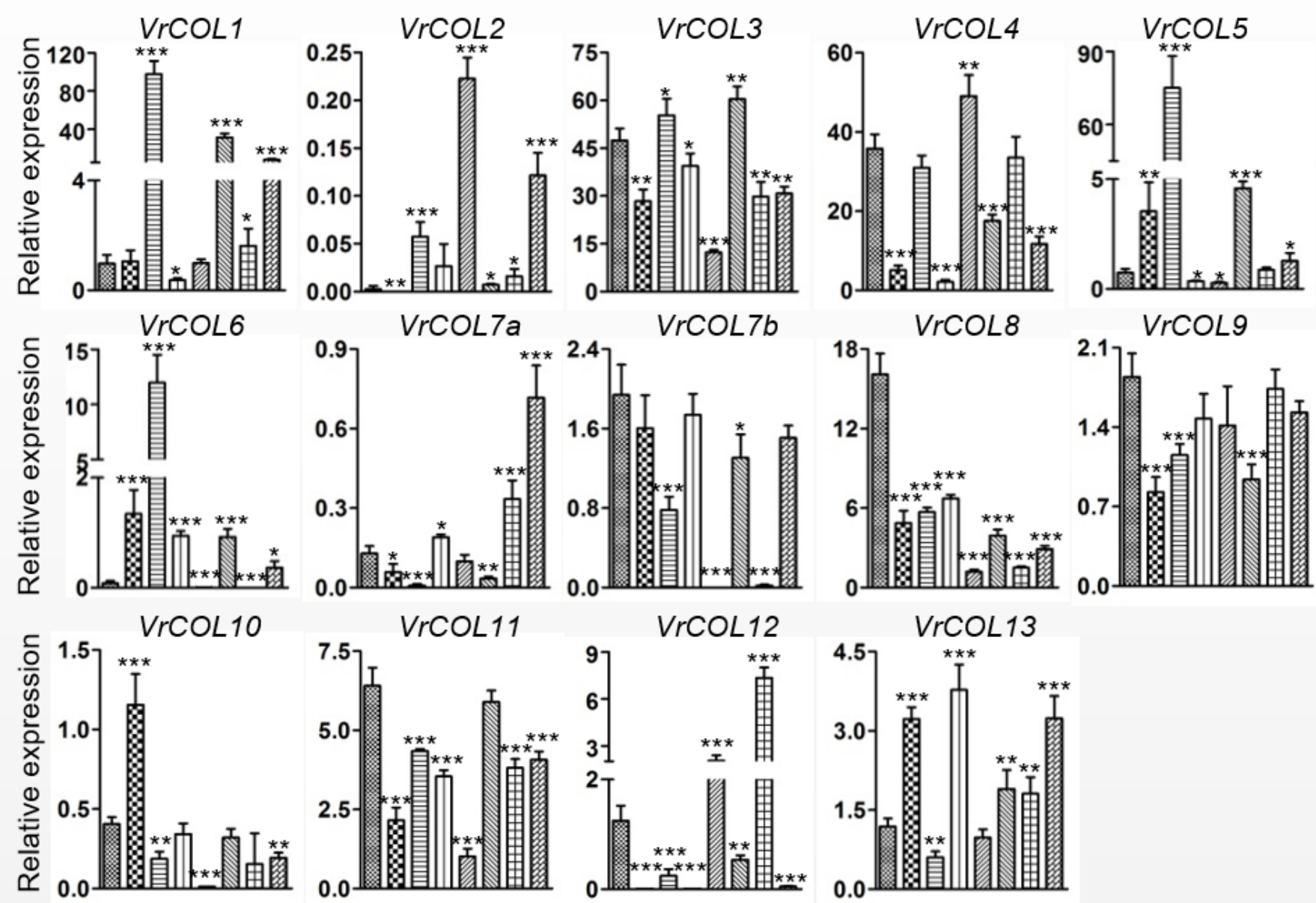

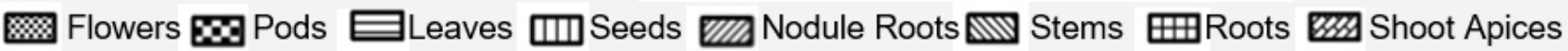

\section{Figure 7}

Relative expression levels of $\mathrm{VrCOL}$ genes in different tissues analyzed by qRT-PCR. The expression level of VrCOL1 in flowers was set as 1 , and the others were adjusted accordingly. ***, ** and * are significantly different at $\mathrm{P}<0.001, \mathrm{P}<0.01$ and $\mathrm{P}<0.05$, respectively, compared with the expression levels in the relative flowers. 

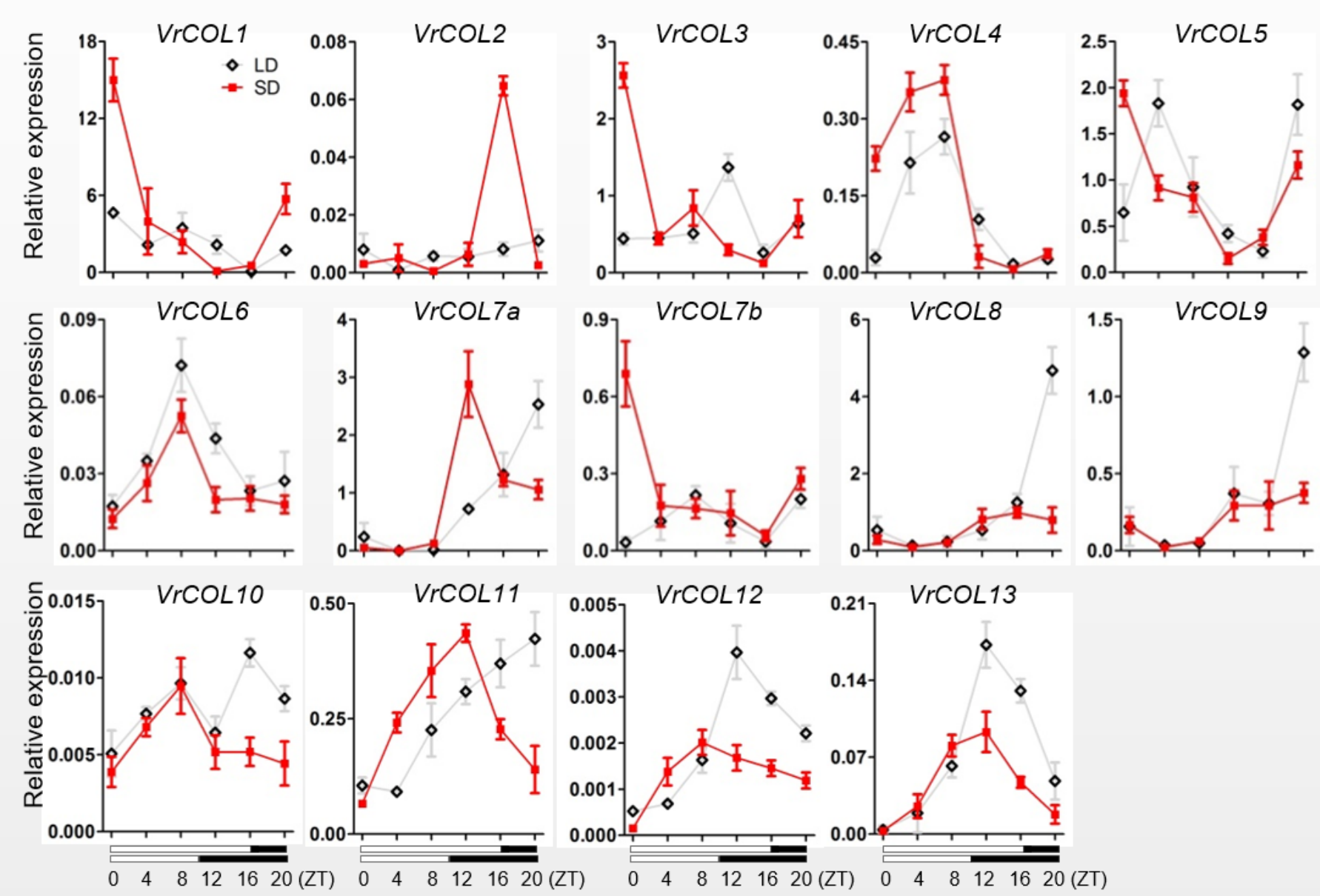

\section{Figure 8}

Relative expression of VrCOLs in leaves throughout the day under SD and LD conditions. The SD condition was set as 8:00 am-6:00 pm light, 6:00 pm-8:00 am dark; the LD condition was set as 8:00 am0:00 am light, 0:00 am-8:00 am dark. ZT, Zeitgeber Time. Expression level of VrCOLs was normalized to an ACTIN gene from mungbean. 

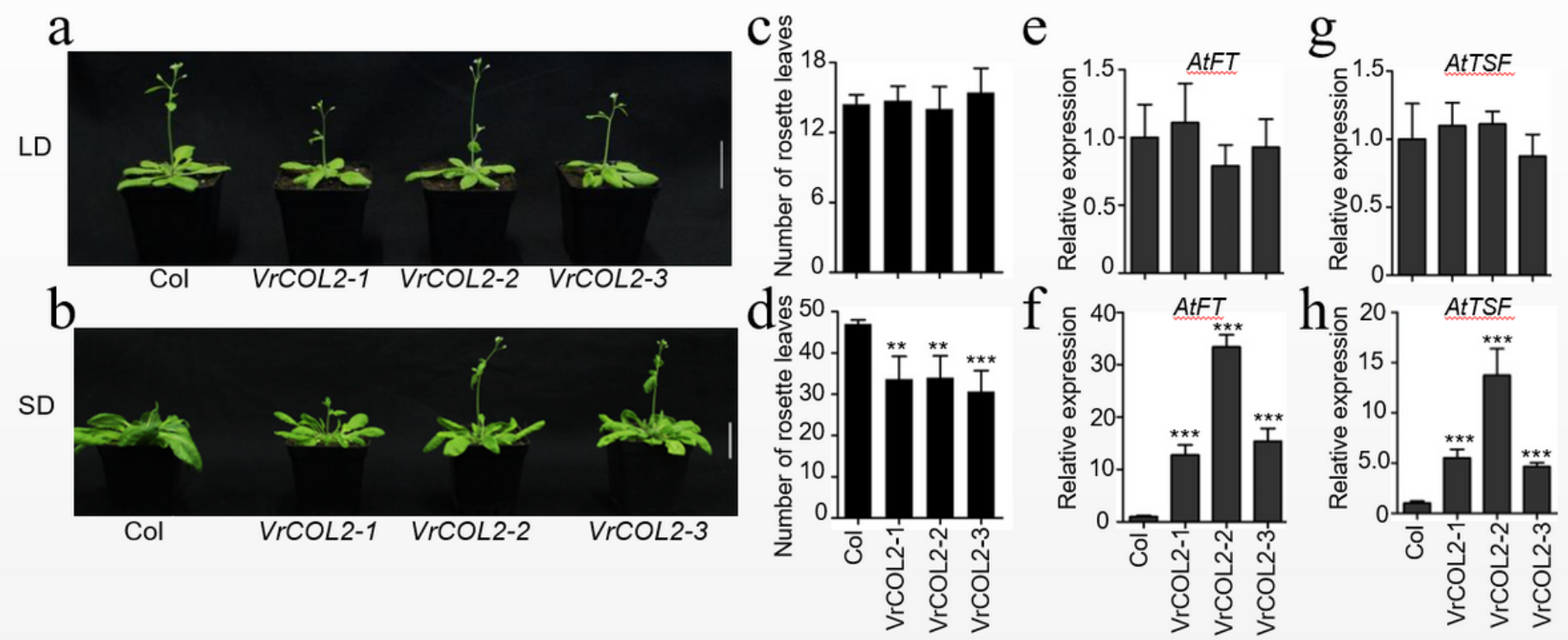

\section{Figure 9}

Overexpression of VrCOL2 accelerates flowering under SD conditions. Phenotypes of $\mathrm{VrCOL2}$ transgenic lines and wild-type A. thaliana (Col) grown under LD (a) and SD conditions (b). The rosette leaf numbers of VrCOL2 transgenic lines and wild-type plants grown under LD (c) and SD conditions (d). The expression levels of AtFT and AtTSF in VrCOL2 transgenic lines and wild-type plants under LD $(e, g)$ and SD conditions ( $f, h)$. A. thaliana leaves were sampled 5 hours after lights-on (ZT 5) from 3-week-old plants. The gene expression in wild type plants was set as 1 and those in other samples were adjusted accordingly. ${ }^{\star * \star} \mathrm{P}<0.001,{ }^{*} \mathrm{P}<0.01$, bars $=4 \mathrm{~cm}$.

\section{Supplementary Files}

This is a list of supplementary files associated with this preprint. Click to download.

- Additionalfile1.pptx

- Additionalfile9.xIsx

- Additionalfile3.xIsx

- Additionalfile5.pptx

- Additionalfile6.pptx

- Additionalfile7.pptx

- Additionalfile4.pptx

- Additionalfile2.pptx

- Additionalfile8.pptx 